\title{
Robust Optimization and Affine Arithmetic for Microgrid Scheduling under Uncertainty
}

\author{
Alfredo Vaccaro \\ Dipartimento di Ingegneria \\ Università degli studi del Sannio \\ Benevento, Italy \\ vaccaro@unisannio.it
}

\author{
Marina Petrelli \\ Dipartimento di Energia \\ Politecnico di Milano \\ Milano, Italy \\ marina.petrelli@polimi.it
}

\author{
Alberto Berizzi \\ Dipartimento di Energia \\ Politecnico di Milano \\ Milano, Italy \\ alberto.berizzi@polimi.it
}

\begin{abstract}
Optimization analyses are commonly used in microgrids to identify the most efficient and reliable operation of the available energy resources. Unfortunately, most of the times these programming problems rely on input parameters which are not accurately known. In this context, advanced computing paradigms for solving uncertainty optimization problems represent the most promising enabling methodology. These techniques may show their effectiveness during both the dispatch and the pre-dispatch phase, when operators need to solve the unit-commitment and the economic dispatch problems. To this aim, this paper discusses and compares experimentally some promising existing alternatives to deterministic methods to deal with the solution of optimization problems in the presence of data uncertainty.
\end{abstract}

Keywords- microgrid scheduling, uncertainty, robust optimization, affine arithmetic.)

\section{INTRODUCTION}

Penetration of microgrids in power systems has been rapidly increasing in the last years, as they can serve multiple purposes in different contexts: in industrialized countries they are mostly employed to power energy-intensive production processes [1], whereas in developing countries they represent one of the main electrification strategies aimed at increasing electricity access [2]. Indeed, microgrid modelling has gained more and more attention, as microgrids come with many issues in terms of security of supply and quality of service. In particular, since nowadays high shares of renewable sources are generally integrated in power systems, the availability of primary resources needs to be taken into account, especially when the microgrid is not connected to the electrical distribution network. In this domain, the uncertainties affecting the input data should not be overlooked, especially in addressing energy dispatch and resource scheduling analyses. Data uncertainties in microgrid modelling stem from multiple and heterogeneous sources, such as the power profiles available from renewable generators, and the load power demand, which represent a complex issue to address especially for isolated systems (i.e. autonomous microgrids) in developing countries, where the behaviour of the customers is more difficult to predict.

Although several approaches have been proposed in the literature for microgrid optimization in the presence of data uncertainty, the selection of the most effective technique is still an open problem, which requires further investigations. Armed with such a vision, this paper aims at presenting and comparing three distinct methods for uncertainty management in microgrid scheduling problems.

The first technique considered in this study is based on a traditional deterministic unit-commitment model, which processes the forecasted values of the uncertain quantities, and tries to identify proper reserve requirements in order to hedge the risks related to the forecast errors of the unpredictable quantities [3], [4]. Recently, alternative and more sophisticated techniques based on a more rigorous formulation of the uncertain optimization problem have been presented in literature. In this context, many papers adopt stochastic methods [5]-[7], with the drawback of requiring information about the probability distributions of the different data instances: these are often difficult to source, especially for applications in developing countries. Therefore, this work employs procedures that do not heavily rely on these pieces of information. The first is robust optimization (RO), in which an uncertainty interval is associated to the uncertain quantities and the algorithm is structured in such a way that the system is protected against any possible realization of the inputs [8], [9]. The other technique tested is affine arithmetic (AA), which is a self-validated computing paradigm that identifies guaranteed enclosures for the computed quantities, considering any uncertainty affecting the input data as well as all internal truncation and round-off errors [10]. The advantage of the latter two approaches is that the user must specify only the extreme values of the uncertainty intervals, without providing any information about the probability distributions, and this is particularly advantageous in case of absence of more detailed data.

The above methods will be compared with reference to a microgrid powered by wind turbines (WTs), photovoltaic (PV) panels and a storage battery bank (SBB), all connected to a DC busbar; back-up diesel generators (DGs) are connected to an AC busbar together with loads. The two busbars are connected through bidirectional converters. On both busbars dump loads are installed to withdraw the energy in excess, which can be present if batteries are already charged and either diesel generators work at their technical minimum, but a lower quantity of energy is requested, or renewable production is higher than the demand. The topology is shown in Fig. 1 and the uncertain quantities are PV production, wind production and loads. The goal of the model is to be as general as possible and to be applicable to most of the real case studies, just by activating or deactivating the different components. Indeed, Fig. 1 constitutes one possible microgrid topology but the formulation benefits from total generality.

The rest of the paper is structured as follows: section II describes the structure of the deterministic algorithm, the objective function and the constraints; section III focuses on robust optimization while section IV gives details on the affine arithmetic approach; the numerical results of the different methods are presented and discussed in section $\mathrm{V}$; finally, conclusions are drawn in section VI. 


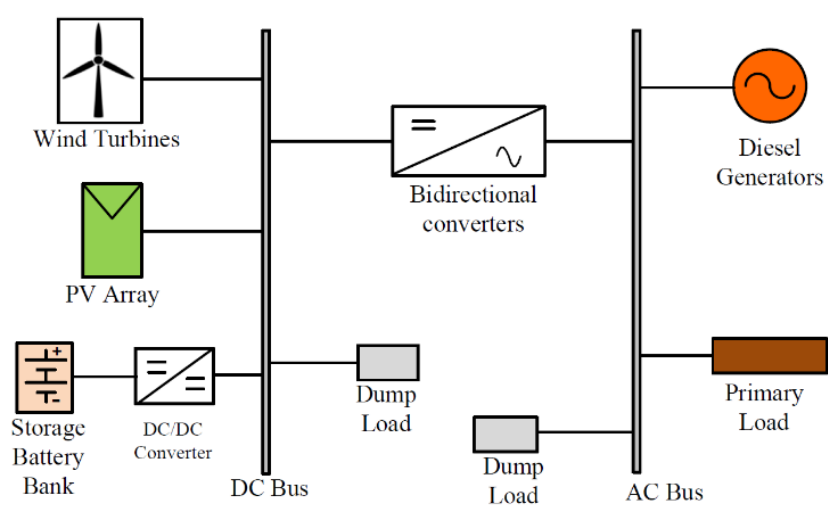

Fig. 1: Plant scheme

\section{DETERMINISTIC OPTIMIZATION}

The purpose of the deterministic model is to define the optimal dispatching strategy of the microgrid. The main variables under the control of the algorithm resolution are the shares of renewable production powering the load or charging the batteries, the diesel production (different types of DGs are available and named $g$ ) and the variables coping with the operation of the battery bank.

The objective function to be minimized is the sum of maintenance and operation costs and it is computed on a 24hours timeframe:

$$
\begin{array}{lr}
\min _{(x, u)} \text { TOCS }=\sum_{h} \sum_{g} C_{f u e l} F C_{h, g}+ & \\
\sum_{h} \sum_{g} U_{h, g} O M C_{g}+\sum_{h} \sum_{g}\left(V_{h, g} S U C_{g}+\right. & \\
\left.Z_{h, g} S D C_{g}\right) & i \in E \\
\text { s.t. } \quad c_{i}(x, u)=0 & i \in I
\end{array}
$$

where TOCS stands for Total Operation Costs of the System. $C_{f u e l}$ is the cost of diesel and $F C_{h, g}$ is the diesel hourly consumption of generator type $g, U_{h, g}$ is the number of online DGs of type $g$ at hour $h, O M C_{g}$ is the operation and maintenance cost for DGs of type $g$. The start-up cost $S U C_{g}$ is considered for the started-up DGs $V_{h, g}$; analogously, the shut-down cost $S D C_{g}$ is considered for the shut-down DGs $Z_{h, g}$. O\&M costs of renewable generators, batteries and converters have been considered to be negligible.

The decision process is subject to a set of constraint functions $c_{i}(x)$, defined in $E$, set of equality constraints, and $I$, set of inequality constraints, with $x$ state variables and $u$ control variables of the algorithm. The scope of the constraints covers technical capabilities of components, balances and physical laws [11].

In particular, the equilibrium of the system is defined by the overall balance constraint (at the AC busbars) that must be fulfilled at any time interval, during which powers are considered to be constant. In addition, there is a number of other constraints describing the balance of the system in order to limit the maximum power flowing through bidirectional converters and to describe the power entering or leaving the battery at each time step. Then, complementarity constraints are needed in order to avoid that converters act as rectifiers and inverters at the same time and that batteries charge and discharge at the same time. Specific constraints are needed for the description of the behaviour of diesel generators: they guarantee that DGs are within their maximum and minimum limit, related to the capability and the performance of the component and to the reserve requirement. Analogously, a block of constraints is specifically devoted to batteries: the operational limits are defined, in order to preserve them and to guarantee the provision of the needed reserve.

To cope with unpredictability in real time, reserve constraints are needed to define the amount of power which must be available hour by hour, according to the unpredictability associated to RES and demand. The same amount of reserve is needed for both upward and downward service and it is provided by batteries and, when online, also by diesel generators:

$$
\begin{array}{ll}
R_{h}^{s y s}=\alpha_{l} \widetilde{D_{h}}+\alpha_{p} \sum_{p} N_{p} \widetilde{P_{h, p}^{p v}}+ & \forall h \\
\alpha_{w} \sum_{w} N_{w} \widetilde{P_{h, w}^{w} t} & \\
\sum_{g} R_{h, g}^{d g}+\sum_{b} R_{h, b}^{s b} \geq R_{h}^{s y s} & \forall h
\end{array}
$$

Equation (2a) sets the total reserve required by the system at hour $h R_{h}^{\text {sys }}$, based on the forecast errors $\alpha_{x}$, with $\widetilde{D_{h}}$ forecasted value of load demand at hour $h, \widetilde{P_{h, p} v}$ forecasted value of production of PV panels of type $p$ at hour $h, \widetilde{P_{h, w}^{w}}$ forecasted value of production of WTs of type $w$ at hour $h, N_{p}$ number of PV panels of type $p, N_{w}$ number of WTs of type $w$. Equation (2b) describes the allocation of $R_{h}^{\text {sys }}$ among DGs $\left(R_{h, g}^{d g}\right)$ and batteries $\left(R_{h, b}^{s b}\right)$.

\section{ROBUST OPTIMIZATION}

The robust model is analogous to the deterministic model, except for the absence of the reserve constraints, as robust optimization treats unpredictability of RES and load by using a different approach: instead of relying on the forecasted values and avoiding to exploit at maximum level the controllable units in order to have reserve available in real time, it includes the uncertainty in the input quantities and guarantees an operation strategy that allows to meet the load in the worst possible case. This is the case of absolute robustness, that for some applications might result too conservative. Therefore, the constraints can be relaxed by means of a parameter $\Gamma$, the budget of uncertainty, which allows the user to tune the conservatism of the model by controlling the total deviation from forecasted values for which the optimization has to be performed [11], [12].

The uncertainty is modelled by the product $\delta_{h, k}^{j} \zeta_{h, k}^{j}$, with $j \in\{L O A D, P V, W T\}, k$ representing the categories to which different uncertainty intervals can be associated (e.g. different technologies of PV panels), where the parameter $\delta_{h}^{x}$ determines the maximum possible uncertainty by which the relevant quantities are affected, while $\zeta_{h}^{j}$ is a variable belonging to the interval $[-1 ; 1]$ and quantifying, in relative terms, the actual shift from the deterministic value. The uncertain quantities can take on infinite different values within the intervals [13]; therefore, the problem needs to be reformulated to become tractable. In order to get a mixedinteger linear programming (MILP) problem, a three-steps procedure is adopted: worst-case reformulation, dual form, robust counterpart.

The first step consists in the formulation of the worst scenario, namely maximum load (i.e. $\zeta_{h, k}^{L O A D}=1$ ) and 
minimum RES generation (i.e. $\zeta_{h, k}^{P V}=-1$ and $\zeta_{h, k}^{W T}=-1$ ), by extracting the expressions of the forecast errors from the original constraints in the global model and solving inner minimization (RES generators)/maximization (load) problems:

$$
\begin{array}{ll}
\min / \max & \sum_{k}\left(\delta_{h, k}^{j} \zeta_{h, k}^{j}\right) \\
\text { s.t. } & \sum_{k}\left|\zeta_{h, k}^{j}\right| \leq \Gamma_{h, j} \\
& \left|\zeta_{h, k}^{j}\right| \leq 1
\end{array}
$$

with $\Gamma_{h, j}$ total budget of uncertainty associated with $j$.

Exploiting the strong duality theorem [12], the dual of each inner optimization problem is formed; its variables have no physical meaning but are associated to the constraints of the primal problem. Finally, the last step consists in the integration of the dual problems into the global algorithm: the objective function of the dual problem needs to be included in the constraint involved in the reformulation procedure, and the constraints of the inner problems must be added to the algorithm. Hence, the robust counterpart of the starting algorithm is obtained, and the model is now tractable as MILP. Details about the all the steps of the reformulation are provided in [14].

\section{AFFINE ARITHMETIC-BASED OPTIMIZATION}

In Affine Arithmetic (AA), each variable is expressed by the sum of a central value and a set of partial deviations, each one describing the variable variation from the central value due to an exogenous source of uncertainty. The latter is represented by a noise symbol, which is a symbolic variable uniformly distributed in the interval $[-1,1]$.

For the considered problem, the input variables are affected by three independent exogenous sources of uncertainty, namely the demand, wind, and load forecasting errors, described by three noise symbols: $\varepsilon_{D}, \varepsilon_{W}$ and $\varepsilon_{P V}$. Hence, the affine forms describing the input variables are:

$$
\begin{array}{lc}
\widehat{D}_{h}=D_{h, 0}+D_{h, 1} \varepsilon_{D} & \forall h \\
\hat{P}_{h}^{p v}=P_{h, 0}^{p v}+P_{h, 1}^{p v} \varepsilon_{P V} & \forall h \\
\hat{P}_{h}^{w t}=P_{h, 0}^{w t}+P_{h, 1}^{w t} \varepsilon_{W} & \forall h
\end{array}
$$

with $\widehat{D}_{h}$ affine form of the demand, $D_{h, 0}$ central value, corresponding to the forecasted value, $D_{h, 1}$ partial deviation; the same stands for $\hat{P}_{h}^{w t, t o t}$ and $\hat{P}_{h}^{p v, t o t}$. Consequently, all the computations involving the input variables should be replaced by the corresponding AA-based operators. To this aim, it is worth noting that the linear operators on real numbers are straightforwardly extended to affine forms, by considering the noise symbols as vector coordinates [15]. These operators preserve the original set of noise symbols, since they do not introduce endogenous uncertainty (i.e. approximation errors), and allow defining equality and inequality operators between affine forms [16].

Thanks to the adoption of these AA-based operators, it is possible to extend the minimization operator for a real-valued function $f(x)$ to an affine function $f(\hat{x})$ :

$$
\begin{aligned}
& \min _{\hat{x}} f(\hat{x}) \rightarrow \\
& \min _{x_{0}, x_{1}, \ldots, x_{n}}\left[f_{0}\left(x_{0}, x_{1}, \ldots, x_{n}\right), \sum_{l=1}^{n}\left|f_{l}\left(x_{0}, x_{1}, \ldots, x_{n}\right)\right|\right]
\end{aligned}
$$

Hence, the minimization of an affine function can be formulated as a deterministic multi-objective problem, and a suitable trade-off between minimizing the central value (i.e. minimization of the nominal value) and the radius, which is the sum of the absolute values of all the partial deviations (i.e. maximizing the robustness to data uncertainty), should be defined by the Analyst [16]. In this work, the adopted solution strategy for multi-objective optimization consists in simply minimizing the sum of the two objective functions. The uncertain decision problem under study can be formulated as follows:

$$
\begin{aligned}
& \min _{(x, u)}\left[f_{0}(x, u)+\sum_{l}\left|f_{l}(x, u)\right|\right] \\
& \text { s.t. } \quad c_{i}(x, u)=0 \quad i \in E \\
& c_{i}(x, u) \geq 0 \quad i \in I
\end{aligned}
$$

with $u$ vector of the deterministic control variables and $x$ vector of the affine forms describing the state variables. As in $\mathrm{RO}$, reserve constraints are not included in the model, since uncertainty management is intrinsic in the formalization of the problem. As an example, the affine form of the total power produced by DGs at hour $h$ is represented in (7):

$$
\begin{aligned}
& \hat{P}_{h}^{d g, t o t}=P_{h, 0}^{d g, t o t}+P_{h, 1}^{d g, t o t} \varepsilon_{D}+ \\
& P_{h, 2}^{d g, t o t} \varepsilon_{P V}+P_{h, 3}^{d g, t o t} \varepsilon_{W}
\end{aligned}
$$

The equality and inequality operators should be replaced by the corresponding affine-based operators, and the objective function to be minimized is the upper bound of the affine function describing the operation costs, whose central value and radius are defined as follows:

$$
\begin{aligned}
& f_{0}(x, u)=\sum_{h} \sum_{g} C_{\text {fuel }} * F C_{h, 0}^{g}+\sum_{h} \sum_{g} U_{h, g} * \\
& O M C_{g}+\sum_{h} \sum_{g}\left(V_{h, g} * S U C_{g}+Z_{h, g} * S D C_{g}\right) \\
& \operatorname{rad}\left(f_{0}\right)=\sum_{h} \sum_{g} C_{f u e l} *\left(\left|F C_{h, 1}^{g}\right|+\left|F C_{h, 2}^{g}\right|+\right. \\
& \left.\left|F C_{h, 3}^{g}\right|\right)
\end{aligned}
$$

The solution of this optimization allows to reliably solve the unit commitment problem by considering all the possible sources of uncertainties affecting the microgrid operation. The obtained solution is robust, since it satisfies all the problem constraints also for the worst-case instance of the input uncertainties. Moreover, the obtained state variables are described by affine forms, which allows to characterize the domains that are guaranteed to include all the expected microgrid states (i.e. solution bounds). Finally, the partial deviations of these affine forms allow to estimate the effect of each source of exogenous uncertainty on the state variables (i.e. uncertainty propagation).

\section{NUMERICAL RESULTS AND DISCUSSION}

In order to compare the two considered approaches to the scheduling of a microgrid, this section presents the results obtained on the real case of a missionary center in the city of Wau, in South Sudan, composed by a radio studio, the house of Comboni nuns, a workshop, the offices of the curia, the residence of the priests and a hospital. The case study has been selected because the data on demand have been reliably collected through accurate field surveys [17]; the inputs on solar and wind power production have been derived from the historical database of Renewable.ninja [18]. These are both the inputs of the deterministic algorithm for photovoltaic and wind generation and the baseline for the uncertain production profiles of the robust and AA model. 
The system is currently powered by 4 independent diesel generators of size $9 \mathrm{~kW}, 12.8 \mathrm{~kW}, 16 \mathrm{~kW}, 32 \mathrm{~kW}$, which are supposed to be integrated, and possibly gradually replaced by renewable generators, namely by $30 \mathrm{~kW}$ of PV, 2 wind turbines of nominal power $10 \mathrm{~kW}$ and about $70 \mathrm{kWh}$ of leadacid batteries. DGs are assumed not to have restrictions in terms of ramps and minimum up- and down-time. For what concerns PV panels, a fixed array (no tracking system) of 1 $\mathrm{kW}$ has been selected, with an $8^{\circ}$ tilt angle. According to the rule-of thumb suggested by [19], it is sensible to choose a unique average tilt angle throughout the whole year, in case the plant is located in a region where solar irradiation does not vary much during the year. Reference [19] suggests it to be simply equal to the latitude of the geographical area taken into consideration. Batteries are modelled with $\mathrm{KiBaM}$ [20] and both charging and discharging efficiency are taken equal to $90 \%$. Finally, converters adopted in the model are characterized by rectification and inversion efficiency of $90 \%$. Input costs are summarized in Table 1.

The deterministic and robust models have been implemented in GAMS 24.0.2 using CPLEX solver, while the AA model has been implemented in Matlab R2014b with standard optimization toolbox. The goal is to elaborate the optimal scheduling for the next day: hence, 24-hours' time snapshots have been used as inputs. Each input value represents the hourly average value of each input during the time interval considered.

Forecast errors for the three uncertain quantities have been defined, but further remarks should be made. In fact, although maximum forecast errors have been set, in some hours they might exceed the deterministic value of the input. In this case, the following procedure has been adopted: when the demand is lower than the maximum error, the amplitude of the uncertainty interval is reduced to a lower value; when the forecasted renewable production is lower than its maximum error (in some hours, it is close to zero), the uncertainty interval is assumed to be null (see Table 2). In particular, as often the case when dealing with developing countries, there was not any information about the variability of the load; therefore, based on background experience, a maximum fluctuation of $3 \mathrm{~kW}$ has been assumed during the day, reduced to $1 \mathrm{~kW}$ during the night. For what concerns renewable production, information about the error was available, differentiated for the two sources. For PV, the outputs are characterized by an RMSE of about 10\% [21]: hence, a maximum error of $0.1 \mathrm{~kW}$ per array sized $1 \mathrm{~kW}$ has been assumed. For what concerns wind production, a study on the

TABLE 1: INPUT COSTS FOR THE OPTIMIZATION

\begin{tabular}{cccc}
\hline $\mathbf{C}_{\text {fuel }}$ & OMC $_{\mathbf{g}}$ & $\mathbf{S U C}_{\mathbf{g}}$ & $\mathbf{S D C}_{\mathbf{g}}$ \\
{$[€ / 1]$} & {$[€ / \mathrm{h}]$} & {$[€]$} & {$[€]$} \\
\hline 0.23 & 0.0161 & 0.01 & 0.01 \\
\hline
\end{tabular}

TABLE 2: MAXIMUM DEVIATIONS FROM FORECAST VALUES

\begin{tabular}{lll}
\hline Demand & when $\widetilde{\mathrm{D}_{\mathrm{h}}}<3 \mathrm{~kW}$ & $\delta_{\mathrm{h}}^{\mathrm{LOAD}} / D_{h, 0}=1 \mathrm{~kW}$ \\
& when $\widetilde{\mathrm{D}_{\mathrm{h}}} \geq 3 \mathrm{~kW}$ & $\delta_{h}^{L O A D} / D_{h, 0}=3 \mathrm{~kW}$ \\
\hline PV & when $\widetilde{\mathrm{P}_{\mathrm{h}, \mathrm{p}}^{\mathrm{pv}}}<0.1 \mathrm{~kW}$ & $\delta_{\mathrm{h}}^{\mathrm{PV}} / P_{h, 0}^{p v}=0 \mathrm{~kW}$ \\
& when $\widetilde{\mathrm{P}_{\mathrm{h}, \mathrm{p}}^{\mathrm{pv}}} \geq 0.1 \mathrm{~kW}$ & $\delta_{\mathrm{h}}^{\mathrm{PV}} / P_{h, 0}^{p v}=0.1 \mathrm{~kW}$ \\
\hline Wind & when $\widetilde{\mathrm{P}_{\mathrm{h}, \mathrm{w}}^{\text {w }}}<1.5 \mathrm{~kW}$ & $\delta_{\mathrm{h}}^{\mathrm{WT}} / P_{h, 0}^{w t}=0 \mathrm{~kW}$ \\
& when $\widetilde{\mathrm{P}_{\mathrm{h}, \mathrm{w}}^{\mathrm{wt}}} \geq 1.5 \mathrm{~kW}$ & $\delta_{\mathrm{h}}^{\mathrm{WT}} / P_{h, 0}^{w t}=1.5 \mathrm{~kW}$ \\
\hline
\end{tabular}

European territory has been made in [22], obtaining quite different results depending on the country, the RMSE being approximately in the interval $5 \div 10 \%$. Taking into account the reduced amount of information available for Africa, RMSE has been therefore supposed to be higher and equal to $15 \%$. Then, since $10 \mathrm{~kW}$ wind turbines are assumed to be installed, the resulting maximum forecast error is $1.5 \mathrm{~kW}$. Finally, the budget of uncertainty, aimed at defining the degree of conservatism of the model, is set so that absolute robustness is reached (i.e., $\Gamma_{h, j}=k$ ) and the system is protected against any possible realization of the inputs.

The results of RO and AA have been made comparable with the outputs of the deterministic model by manipulating the reserve constraints so that the total reserve available is as close as possible to the total uncertainty associated to the inputs in RO and AA optimization. As shown in Fig. 2, it is impossible to get to a perfect superposition because the two elements are defined in different ways: the reserve is modelled in such a way that it changes hour by hour, according to the actual demand and RES production level (see constraint (2a)); the total uncertainty of RO and AA is instead tailored on the size of RES generators and on maximum demand. Additionally, further values of maximum forecast errors have been defined for the hours in which quantities are low. Therefore, two steps have been set for each $\delta_{h, k}^{j} /$ central value (see Table 2) and the total uncertainty evolves through discrete variations. The result shown in Fig. 2 has been obtained by manipulating the forecast error coefficients of (2a), in order to obtain a total reserve profile which could resemble as much as possible the total uncertainty curve, using reasonable figures. The final values of $\alpha_{l}, \alpha_{p}, \alpha_{w}$ are $25 \%, 15 \%$ and $40 \%$ respectively.

As shown in Fig. 3, the three techniques provide different scheduling strategies. Both deterministic and AA optimization employ DGs for 3 hours per day; moreover, they both use the energy stored in batteries during the first half of the day to power the load during the afternoon and then return to the initial state of charge thanks to the energy provided by the wind turbines. The worst-case RO, not surprisingly, produces a more conservative scheduling, namely a much more intensive use of DGs and a smoother operation of batteries; renewable energy is completely exploited. These results lead to a significantly higher operation cost in the case of RO (see Table 3).

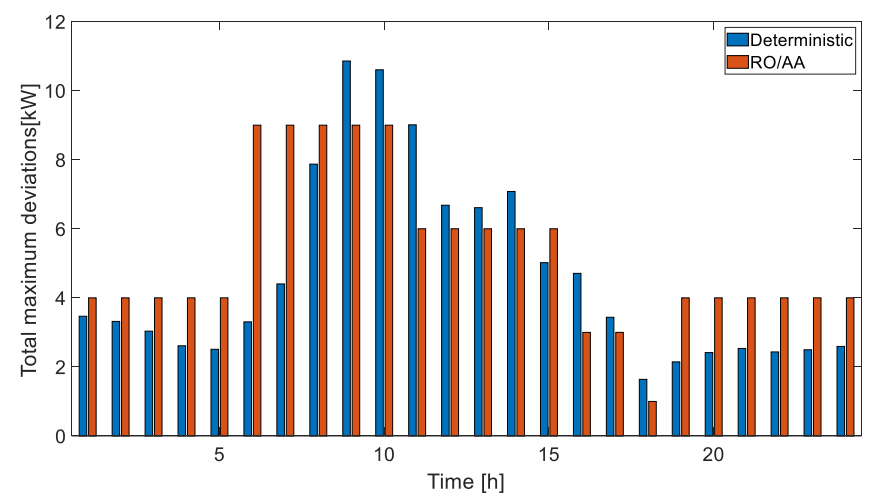

Fig. 2: Comparison between total reserve provided in the deterministic approach and total deviations of inputs form forecasted values in robust and AA model 

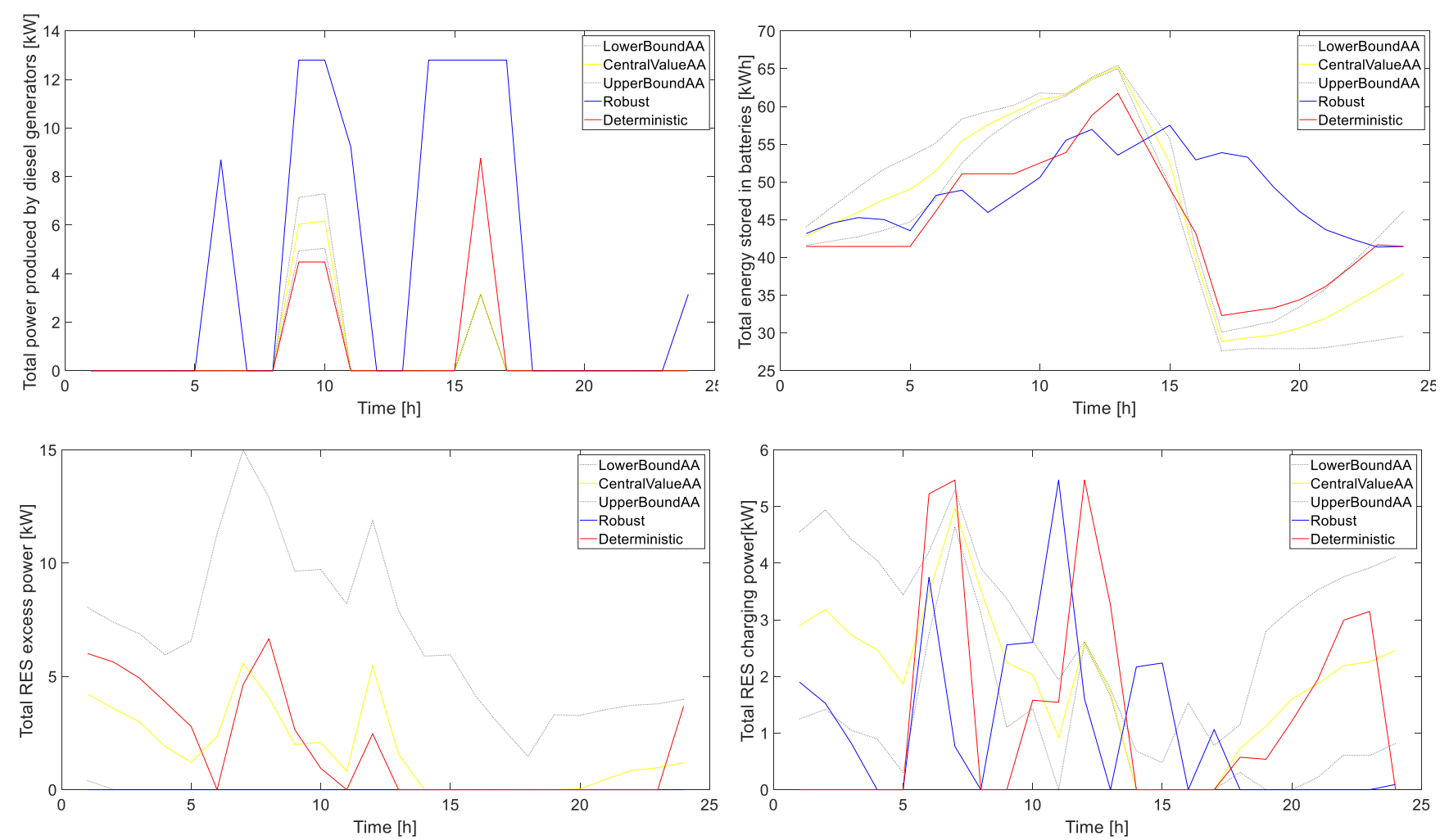

Fig. 3:Daily scheduling of resources according to the deterministic, robust and AA optimization

The crucial element determining the relevant difference of the output lays in the different approach in treating inputs. In the deterministic optimization, forecasted values of load demand and RES availability are used to define the operation of programmable resources, which need to be available to provide the scheduled reserve in case of unbalance (i.e. deviation from forecasts in real time). AA produces similar results, as the forecasted quantities constitute the central values of the input variables. For what concerns RO, the reformulation procedure, needed to make the problem tractable as MILP, produces a new deterministic problem to be solved in the worst-case scenario. Less RESs are available and more power is requested to the system; therefore, DGs are often employed during the day. The advantage of the RO proposed is the presence of the budget of uncertainty, that can be easily tailored on the case under study according to the desired conservatism of the results.

Hence, RO provides a scheduling strategy that guarantees the targeted continuity of supply while AA gives more flexible results, suitable for less demanding systems.

Finally, it is worth observing as in AA all the control variables of the optimization problem are expressed by affine forms, which allow the analysts to obtain valuable information about the expected microgrid operation state. For example, by analyzing the affine form (7) at hour 9:

$$
\begin{aligned}
& \hat{P}_{9}^{d g, t o t}=5.9618+1.1523 \varepsilon_{D}-0.0081 \varepsilon_{P V}+ \\
& 0.0037 \varepsilon_{W}
\end{aligned}
$$

much information can be inferred. First, the generated power is guaranteed to vary in the range [4.7977, 7.1259] $\mathrm{kW}$, considering all the possible instances of the input uncertainties. Second, the generated power deviates from the nominal value of $5.9618 \mathrm{~kW}$ due to the effect of the load $( \pm 1.1523)$, photovoltaic $( \pm 0.0081)$ and wind $( \pm 0.0037)$ forecasting uncertainty, respectively. This allows estimating the impact of each source of input uncertainty on the control variables, hence supporting the development of large-scale sensitivity analysis. Lastly, during real-time operation, when the uncertain input data degenerate to deterministic variables, the optimal generators dispatch can be computed as:

$$
\begin{aligned}
& \mathrm{P}_{9}^{d g, t o t}=5.9618+1.1523 \bar{\varepsilon}_{D}-0.0081 \bar{\varepsilon}_{P V}+ \\
& 0.0037 \bar{\varepsilon}_{W}
\end{aligned}
$$

with the noise symbols derived from:

$$
\bar{\varepsilon}_{D}=\frac{\mathrm{D}_{h}-\mathrm{D}_{h, 0}}{\mathrm{D}_{h, 1}} ; \bar{\varepsilon}_{P V}=\frac{\mathrm{P}_{\mathrm{h}}^{\mathrm{pv}}-\mathrm{P}_{\mathrm{h}, 0}^{\mathrm{pv}}}{\mathrm{P}_{\mathrm{h}, 1}^{\mathrm{pv}}} ; \bar{\varepsilon}_{W}=\frac{\mathrm{P}_{\mathrm{h}}^{\mathrm{wt}}-\mathrm{P}_{\mathrm{h}, 0}^{\mathrm{wt}}}{\mathrm{P}_{\mathrm{h}, 1}^{\mathrm{wt}}}
$$

\section{CONCLUSIONS}

Efficient and reliable operation of autonomous microgrids require the deployment of advanced techniques for representing and managing the multiple and heterogeneous sources of uncertainty affecting the input data. To address this complex issue, three computing paradigms for uncertain optimization analyses have been presented, and their performances have been assessed and compared experimentally on a realistic case study.

The results obtained show that an analyst aiming at solving uncertain microgrid programming problems is confronted with an accuracy/complexity trade-off. On one side, there are solution techniques based on conservative reserve allocation, which offer a rough qualitative insight of the solution in a very

TABLE 3: OPTIMIZATION RESULTS

\begin{tabular}{lccc}
\hline & $\begin{array}{c}\text { Deterministic } \\
\text { optimization }\end{array}$ & $\begin{array}{c}\text { Robust } \\
\text { optimization }\end{array}$ & $\begin{array}{c}\text { AA } \\
\text { optimization }\end{array}$ \\
\hline TOCS [€] & 1.309 & 5.958 & $1.2390 \pm 0.035$ \\
\hline $\begin{array}{l}\text { Computational } \\
\text { burden [s] }\end{array}$ & 0.422 & 0.266 & 1.688 \\
\hline
\end{tabular}


short time. On the other side, there are affine arithmetic and robust optimization-based methods that return a more reliable description of the uncertainty evolution; this comes at the cost of a longer simulation time in the AA case and of the sensitive definition of the most suitable budget of uncertainty in the RO case, that heavily influences the resulting operation costs.

The Authors are confident that the obtained results would help making the choice once information about the use of the simulation output, the available computing resources and the maximum allowable risk are available.

A future possible development of this study is the comparison of the three methods on the design phase of a microgrid, for which computational burden and accurate modelling are much more challenging already in the scope of deterministic optimization and for which the weight of uncertainty on the results is much heavier, as extremely long timeframes are taken into consideration.

\section{REFERENCES}

[1] H. B. Cetinkaya, S. Kucuk, M. Unaldi, and G. B. Gokce, "A case study of a successful industrial microgrid operation," 2017 4th International Conference on Electrical and Electronics Engineering, ICEEE 2017, pp. 95-98, 2017.

[2] J. P. Murenzi and T. S. Ustun, "The case for microgrids in electrifying Sub-Saharan Africa," pp. $1-6,2015$.

[3] A. M. Zein Alabedin, E. F. El-Saadany, and M. M. A. Salama, "Generation scheduling in Microgrids under uncertainties in power generation," 2012 IEEE Electrical Power and Energy Conference, EPEC 2012, pp. 133-138, 2012.

[4] A. Zakariazadeh and S. Jadid, "Energy and Reserve Scheduling of Microgrid Using Multi-Objective Optimization," in 22nd International Conference and Exhibition on Electricity Distribution (CIRED 2013), 2013, pp. 3-6.

[5] W. Su, J. Wang, and J. Roh, "Stochastic energy scheduling in microgrids with intermittent renewable energy resources," IEEE Transactions on Smart Grid, vol. 5, no. 4, pp. 1876-1883, 2014.

[6] M. Alipour, B. Mohammadi-Ivatloo, and K. Zare, "Stochastic Scheduling of Renewable and CHPBased Microgrids," IEEE Transactions on Industrial Informatics, vol. 11, no. 5, pp. 1049-1058, 2015.

[7] N. Liu, M. Cheng, X. Yu, J. Zhong, and J. Lei, "Energy-Sharing Provider for PV Prosumer Clusters: A Hybrid Approach Using Stochastic Programming and Stackelberg Game," IEEE Transactions on Industrial Electronics, vol. 65, no. 8, pp. 6740-6750, 2018.

[8] H. Zhang, D. Yue, and X. Xie, "Robust Optimization for Dynamic Economic Dispatch under Wind Power Uncertainty with Different Levels of Uncertainty Budget," IEEE Access, vol. 4, pp. 7633-7644, 2016.

[9] S. Li, J. Wen, J. Li, X. Han, and F. Jiakun, "Coordinated optimization of wind generation and pumped-storage plant by robust unit commitment," 2016 IEEE International Energy Conference, ENERGYCON 2016, 2016.

[10] D. Romero-Quete and C. A. Canizares, "An Affine Arithmetic-Based Energy Management System for
Isolated Microgrids," IEEE Transactions on Smart Grid, vol. 3053, no. JANUARY, pp. 1-11, 2018.

[11] G. G. Moshi, C. Bovo, A. Berizzi, and L. Taccari, "Optimization of integrated design and operation of microgrids under uncertainty," 19th Power Systems Computation Conference, PSCC 2016, 2016.

[12] D. Bertsimas and M. Sim, "The Price of Robustness," Operations Research, vol. 52, no. 1, pp. 35-53, 2004.

[13] B. L. Gorissen, I. Yanikoğlu, and D. den Hertog, "A practical guide to robust optimization," Omega, vol. 53, pp. 124-137, 2015.

[14] M. Petrelli, A. Berizzi, C. Bovo, and E. Amaldi, "Robust optimization for the scheduling of isolated RES-based microgrids in developing countries," in MEDPOWER2018, 2018.

[15] A. Vaccaro, C. A. Canizares, and D. Villacci, "An affine arithmetic-based methodology for reliable power flow analysis in the presence of data uncertainty," IEEE Transactions on Power Systems, vol. 25, no. 2, pp. 624-632, 2010.

[16] A. Vaccaro and C. A. Canizares, "An Affine Arithmetic-Based Framework for Uncertain Power Flow and Optimal Power Flow Studies," IEEE Transactions on Power Systems, vol. 32, no. 1, pp. 274-288, 2017.

[17] G. G. Moshi, M. Pedico, C. Bovo, and A. Berizzi, "Optimal generation scheduling of small diesel generators in a microgrid," ENERGYCON 2014 IEEE International Energy Conference, pp. 867-873, 2014.

[18] "Renewables.Ninja." [Online]. Available: https://www.renewables.ninja/.

[19] L. Castañer, S. Bermejo, T. Markvart, and K. Fragaki, "Energy Production by a PV Array," in Practical Handbook of Photovoltaics, Elsevier Ltd, 2012, pp. 645-658.

[20] E. I. Vrettos and S. A. Papathanassiou, "Operating Policy and Optimal Sizing of a High Penetration RES-BESS System for Small Isolated Grids," IEEE Transactions on Energy Conversion, vol. 26, no. 3, pp. 744-756, 2011.

[21] S. Pfenninger and I. Staffell, "Long-term patterns of European PV output using 30 years of validated hourly reanalysis and satellite data," Energy, vol. 114, pp. 1251-1265, 2016.

[22] I. Staffell and S. Pfenninger, "Using bias-corrected reanalysis to simulate current and future wind power output," Energy, vol. 114, pp. 1224-1239, 2016. 